\title{
Using Competitive Intelligence to Develop an Automated Visa Approval System
}

Katherine M. Shelfer and June M. Verner

Drexel University

Philadelphia PA

kathy.shelfer@cis.drexel.edu

\begin{abstract}
The use of competitive intelligence techniques identified the need for an automated visa approval system to streamline straightforward visa decisions that allows analysts more time for problematic applications. Such a system facilitates the capture and use of lessons learned and encourages knowledge sharing to support global terrorism risk management. However, automation of visa approvals raises issues related to human rights, data quantity and quality, choice of models and the correct interpretation of contextualized results.
\end{abstract}

Keywords: authentication, competitive intelligence; crime; information systems; identification fraud; models; risk management; terrorism

\section{BACKGROUND AND INTRODUCTION}

This paper establishes the framework for the development of an integrated automated visa approval system (AVAS). It discusses the use of corporate systems to improve automated decision support for the visa approval process, and highlights the role that competitive intelligence can play in strategic identification, acquisition and management of relevant knowledge. Visa risk assessment begins with authentication of individuals and requires integration with other decision support systems.

eGovernment is primarily concerned with carrying out government business transactions electronically, usually over the Internet, but it includes all related real-world processes. eCommerce has paved the way for eGovernment and acts as a catalyst for the implementation of eGovernment strategies for several reasons. First, customers have competing demands for their time, so they want to be able to decide when (and how) to optimize 
their choices. The Internet can be used to offset some of the limitations of "bricks and mortar" access to government services. Second, eCommerce successes indicate that it may be possible to improve decision support when operations have similar requirements. There is much speculation about where eGovernment is going and what it will mean for all of us in the future [O'Neill 2000]. One certain direction is computerization of visa applications and the automated integration and use of corporate and government data to improve decision support for the visa approval process. This will reduce the burden on overworked visa analysts. We discuss this further below.

To improve the visa approval process without automation gives us several possible solutions, including (1) hiring and training more analysts and managers, or (2) slowing down the approval process to respond to public pressure for better decisions. A study of these potential solutions results in awareness that the best option is to automate the "easy stuff." This would reduce costs, protect staff and save time for complex or suspicious casesthe "exceptions" that require human judgment. In others words, a strategic implementation of a knowledge management system to support visa risk assessment is the fastest and most cost-effective alternative. An advantage of this approach is that it should be possible to capture and share useful insights and improve the expertise of analysts, transitioning them from bottlenecks to facilitators. However, the ability to take appropriate action depends on a clear understanding of the problem.

In constructing a visa approval system to support global risk assessment, three issues are inextricably linked through the ability to authenticate individuals: national security, critical infrastructure and commerce. However, the basic issues are the same ones that corporations and governments generally face when they begin to develop any integrated information system. Besides accurate user requirements specification, there are a number of problems related to data cleansing, project management and technical issues. The major human issues to be addressed focus on:

- agreement of the exact system requirements;

- facilitation of information sharing and reduction of knowledge hoarding;

- establishment of physical security for facilities and systems; and

- enforceable (and enforced) records management and privacy protocols.

When we think of global risk assessment, especially for a visa approval system, the difference between eCommerce and eGovernment systems is the unprecedented need to share accurate and relevant personal information on individual citizens and on corporate and individual transactions (such as financial activities) between nations. Any such effort must safeguard legal protections and respect national boundaries, yet facilitate early detection and entry prevention of terrorists and their sponsors. It would also be helpful to be able to identify the profiles of those who are responsible for the inflow 
and outflow of money used to fund terrorist activities. The differences between corporate and government systems are primarily related to the need to carefully balance between two imperatives: (1) protecting the human rights of individuals and (2) preventing mass victimization by terrorists who wish to destroy the human rights of others while claiming such protections for themselves. For this reason, developing a state of the art visa approval system is an important focus of a global risk assessment technology initiative.

Automated risk assessment systems must employ advanced algorithms with large numbers of combined data sets to determine the statistical probability of "intent to harm." The dangers of improperly developing and using such as system cannot be overemphasized. There are obvious concerns about (1) human rights, including individual privacy, (2) data quantity and quality; and (3) establishing and correctly interpreting parameters for modeling and scoring of an individual's risk. For this reason, (1) legal and ethical frameworks must be embedded into the models and (2) analysts must be core members of the project team from the outset.

The visa decision process is usually paper-based. There is very little secondary evidence to support the decision. The number of applicants, the geographic dispersion at the intake stage, and the need to be familiar with a large number of conditions has overwhelmed visa analysts. The current situation is not the result of stupid analysts making bad decisions, but of a lack of opportunity and incentive to share data that might be important in the decision to approve or deny a visa in another location. There is also a lack of easily accessible archival data and there are insufficient budgets for information and information technology that might rectify this situation. As a result, the system does little to support the prediction of an individual's future behavior as either benign or representing an intent to harm others.

Automating the "easy" decisions might support more effective communication between analysts and more

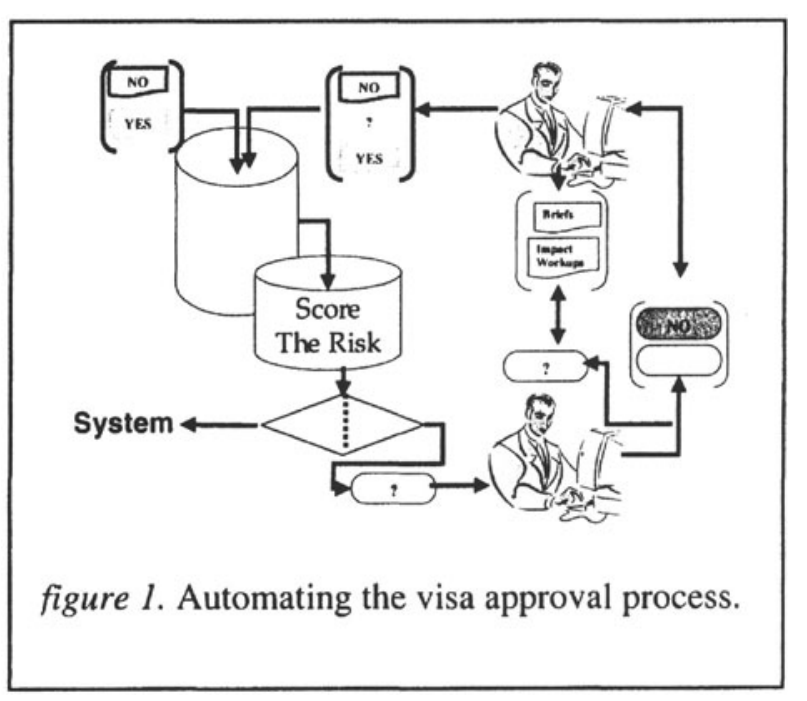
effective use of their time (see figure 1). By reducing the handling (and the time drain) of the many "easy" decisions, analysts should be able to focus more closely on the "exceptions" that require human judgment. The capability to capture and mine historical data allows analysts to benefit from shared expertise and improved insight over time can lead to operations that are more efficient and more effective. Reduced hardware and processing 
costs can lower the costs of providing the same basic government services to the many thousands who apply for visas each business day. Analysts might also be able to communicate more effectively across distances and time zones.

Planning for an AVAS involves a number of related concerns that are discussed in the following sections: human rights issues, data quantity and quality, competitive intelligence; credit modeling/scoring for decision support; and choosing and validating data mining models.

\subsection{Human Rights Issues, Data Quality and Quantity}

There are two major human rights concerns at the individual level: (1) misidentifying the innocent individual as a potential terrorist, and (2) failing to identify the terrorist. The unfamiliar is often considered suspicious in times of crisis, so it is important to develop systems that examine patterns and processes in an equitable fashion. This is mission critical because, though the names and the faces of the players may change, the patterns and processes (often overlapping with the modalities of other forms of crime) serve to signal intent to harm and are more likely to remain stable over a longer period.

There are two major issues related to the required data: quantity and quality. Both of these issues are related to our need to answer the following questions: Do we know what we need to know? How should we authenticate the individual applicants? How do we verify any of the data on this application? Who else should we ask? Are we asking visa applicants the right questions? Are the responses from applicants useful? Is there anything else we should be asking? If so, what might that be? How much data do we need? Where will we obtain it? How long should we keep it? And in what form?

Data Quantity. Because there is a problem of scale in accepting and processing visa applications, the average visa application receives very little attention before a decision is made. Governments must depend heavily on overworked analysts and an applicant's innocence of intent to harm. Very little data on past decisions is in electronic form. Until recently, the cost of digitizing and processing these records was prohibitive. Unfortunately, the price of failure to improve the system is potentially substantial harm.

Data Quality. The unavoidable truth is that currently visa applicants are asked some of the right questions, but they are not asked for everything that is relevant. The answers given by applicants may be neither truthful nor accurate. Relevant information may not be shared among analysts. Shared information may not be timely. Interpretations of what is "meaningful" may also change over time and through context. For this reason, it is best to start with incremental improvements to small systems, such as applications for 
flight school training, before moving to a larger system, such as one for visa approvals. Lessons learned from improving a smaller system can be used to guide the development of larger systems and eventually support integrated global risk assessment.

\subsection{Competitive Intelligence}

Given the complexity of the challenge, it is imperative to use Competitive Intelligence (CI) techniques to develop and deploy integrated information systems [Shelfer, 2002]. CI is defined as the legal collection and analysis of information regarding the capabilities, vulnerabilities, and intentions of business competitors, conducted by using information databases and other "open sources" and through ethical inquiry. ...a continuous process [that includes] analysis that does not avoid unwelcome conclusions, and controlled dissemination of actionable intelligence to decision-makers. By analyzing rivals' moves, CI allows companies to anticipate [and shape] market developments rather than merely react to them [SCIP 2001]. The multidisciplinary nature of competitive intelligence enables practitioners to draw from a wide range of analytical methods. The two discussed in this paper are (1) Key Intelligence Topics (KITs) [Herring, 1999] and Gap Analysis, a component of benchmarking [Gap, 2001-2002]. The goal of gap analysis is to measure existing capabilities against desired outcomes and use this new insight to plot changes in direction and rate of change. The size of the development effort required to assess global risk and the importance of time to completion indicates that gap analysis would be helpful in identifying and prioritizing incremental development efforts.

Using CI techniques to support strategic knowledge management enables an organization to optimize information and convert it to insights. These insights can be used to position an organization to effectively and efficiently respond to external opportunities and threats. The point of origination for this evolution is the knowledge base. Data that is used to populate such knowledge bases is of four types: (1) demographic, (2) psychographic, (3) geographic, and (4) political/ administrative. Examples of political/administrative data include laws, policies, regulations, and standard procedures. Where data from a variety of sources must be integrated and used for decision support, a system is designed based on detailed user needs assessments using formal requirements elicitation methods. This results in specific guidelines for technical design and system integration. Once the system is in place and the knowledge base has been populated, the data can then be mined [Thearling 2002]. 
The process of knowledge discovery involves the development and testing of models for their predictive value (see figure 2). Once the models have been tested, the results are converted to scores and the scores are interpreted and categorized. The major benefits include information reports and (2) decision support (model and score). By automating the clear decisions that respond to standard criteria, analysts gain time for exception processing-

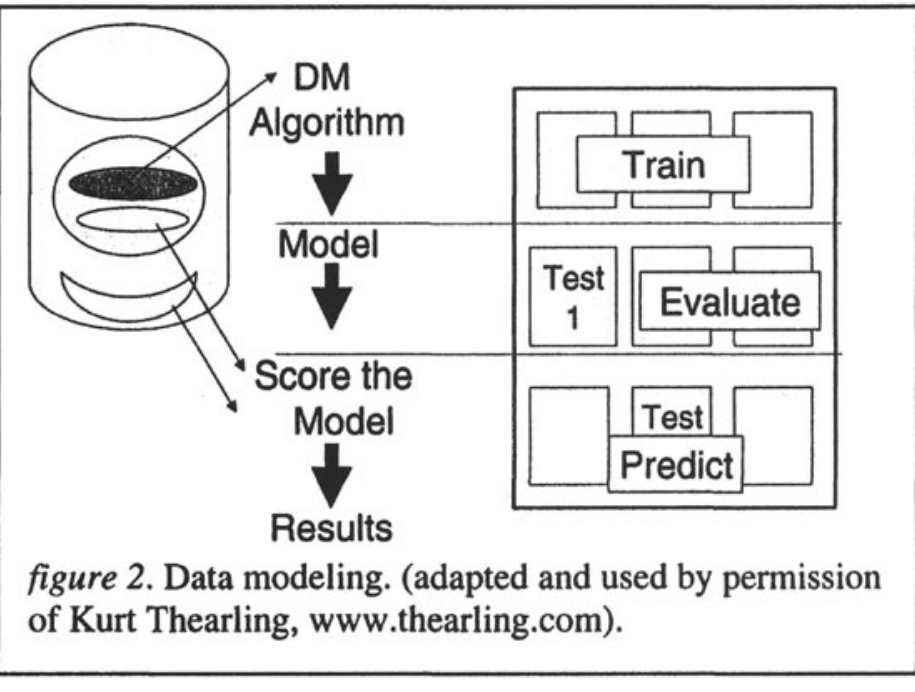
the "active listening" required if they are to successfully perform their duties in a noisy information environment.

Analytical models use existing categorical data and past outcomes to improve prediction of future actions. Models (1) optimize similarities (improve mean response time) and (2) guide discovery (identify specific indicator variables, examine combinations and clusters of variables, and reduce the background noise). Because models can also be used to test our assumptions, they can improve risk assessment by identifying nonlinear variables, and clusters of variables that are predictive, especially during those times when information clutter or personal biases hinder human analysts. The scoring engine then provides the framework for interpretation of the results of using the model to filter the data.

Using gap analysis, we find that the most sophisticated systems for risk assessment appear to be the proprietary systems used for credit modeling and scoring that embed tools for spotting identification (ID) fraud. Such credit scoring systems are roughly analogous to approving visas for large numbers of individual (unknown) applicants. For this reason, many of the features and capabilities of credit scoring systems that embed ID fraud detection can be used to acquire the relevant applications and expertise needed to develop the new integrated visa approval system. By standardizing on such existing systems, organizations are better able to (1) protect themselves from civil or criminal liability; (2) promote integrity and customer confidence; and (3) meet or exceed legal and regulatory obligations. According to Kervisa, the nature of the visa approval process involves many of the targeted legal/regulatory issues associated with markets that require due diligence and are plagued by ID Fraud. [RiskWise ${ }^{\mathrm{TM}}, 2002$ ].

There are several factors that involve fraud detection. First, market forces demand the refinement of risk assessment. Since the low-risk credit 
markets are saturated, competitors must improve their ability to identify and approve credit for good risks by "bottom fishing" in high-risk markets. To reduce their losses, they must also deny credit to bad risks in low-risk markets. Second, credit risk is partly a factor of timing. Fraudulent applications are more likely to slip through the cracks when analysts are overworked during an avalanche of applications. Third, ID fraud, which is facilitated by the use of the Internet, plays a large part in a wide range of crimes [Willox, 2002]. One example is economic crime, including money laundering, which provides terrorists with access to funds.

eCommerce success also depends on strategic knowledge management. A synthesis of insights taken from www.brint.com focuses on strategic knowledge management and the refined use of business intelligence to improve decision support. This involves the explicit and systematic creation, management and use of knowledge gleaned from the capture and use of the information and accumulated wisdom of others. In the specific sense used in this paper, strategic knowledge management requires the creation, development and discovery of intelligence that supports smarter decisions for visa approvals/denials. To accomplish this goal, an automated visa approval system would combine data mining (modeling and scoring for risk assessment) with Case-Based Reasoning (CBR) and Lessons Learned Systems (LLS). This would enable collaborative use of shared insights to become part of the visa approval system. By organizing data in a useful fashion, ambiguity is reduced and insight is improved.

Automating the visa approval system would help to (1) identify potential risk events and (2) manage assumptions. It would support analysis and response to potential threats. Knowledge Management is also about the efficient and effective generation, storage, and sharing of information, and about how fast an organization can leverage that knowledge. [Why eGovernment, 2002]. For any such process to be a success, however, leaders must (1) identify clear objects with defined benefits, (2) embed sharing into the culture of creation and use, and (3) develop a workable information architecture that supports iteration and refinement of results. Knowledge management tools can help to identify "unmanaged assumptions" and provide more information about how either the customer or the service provider acquires, structures, stores, and/or distributes knowledge to those who need it. This will enable us to successfully complete a visa approval system development initiative on time and within budget.

CI practitioners are particularly useful in this effort because they (1) are comfortable with ambiguity, (2) understand issues related to interpretation of "dirty" data, and (3) look for the deeper implications and potential impacts of current choices on future performance. The choice of a model depends squarely on understanding the requirements. In a visa approval system, like the credit decision, the need for quick approval must be balanced with the need to correctly deny access (and opportunity) to those who wish to harm 
others. One useful process that $\mathrm{CI}$ practitioners use is the Key Intelligence Topic (KIT) that takes a topic and develops a series of underlying questions (KIQs) that are straightforward (and often binomial) in nature. Answering these KIQs provides the insight required to develop actionable about the KIT. For example, a KIT can be used to identify appropriate data mining and data modeling techniques and a KIT can guide comparative evaluations of vendors in a range of industries that develop and supply these solutions. KITs can also be designed to identify important issues in similar settings that deal with the need to manage expectations when the price of being wrong may be too high to allow the number of "defects" that would be "acceptable" in a typical model. Major issues that should be addressed include:

- Models designed for one subset are not reliable when used to score a different subset;

- Assumptions and contexts change over time, and can damage the reliability of the results;

- All of the data fields used as input to the model should be available for scoring (not all fields used to build the model are needed to score the model); and

- Data inputs should match data fields in the model.

Before a model can be constructed, the focus must be clear-will this model support the entire visa approval system or some subset, such as the "26 hot spots" of immediate concern? For this reason, the choice of an underlying database to use in constructing and testing the models is of paramount importance (see figure 1, above). A typical credit scoring system has an advantage over a newly automated visa operation, in that there is a large warehouse that serves as a testing environment. For AVAS, the equivalent would be all the visas ever approved. The data mart would be one or more subsets of interest such as all foreign nationals who are applicants for flight schools. While this might appear to be closing the door after the horse has bolted, it is a useful exercise. In fact, the difficulty related to developing a valid model for a visa risk assessment system is that there is very little past data available on which to build and test predictive models. A test case of 19 middle eastern males involved in three facets of a single action is not statistically valid as a predictor. However, we can use lessons learned from combining insights of others to isolate the highest risk and the lowest risk behaviors, which enables us to improve our awareness of "Mr. /Ms. Good Risk" and "Mr. /Ms. Bad Risk." 


\subsection{Credit Modeling/Scoring for Decision Support}

The underlying computational methodology of a global risk assessment system requires the development of a set of quantitative models that can be used to improve our ability to manage risk. As these models are refined through use over time, they can improve the speed and accuracy of global risk assessment. Research has demonstrated that discriminatory bias is reduced when decisions are quantified using predefined criteria based on pattern analysis of large data sets. Credit Scoring systems are the most sophisticated of such technologies. There are at least four major types of credit-scoring models:

- Credit Flow --tighten/loosen the flow of capital

- Crowding Out - assumes that government is not discriminatory, then estimates credit choices

- Default (Failure) rates - uses past data to predict future failure rates

- Rejection Probability models - uses data from individual applications

Models are intended to isolate actual events on which interpretations can be based. For this reason, one of the most beneficial aspects of credit scoring has been a documented and verified reduction in discriminatory credit practices in high-risk markets. This is an important benefit of an automated process as politically sensitive as visa risk assessment.

As noted earlier, in the rush to extend credit, many financial services firms have failed to take into account the proportion of "bad risks" in the high-income pool and have ignored the presence of "good risks" in the subprime markets. The need to improve decision support in order to safely grow their market shares requires that financial services isolate the worst of the risks for denial and extract the best of the "worst" for approvals. In the process of due diligence, one of the key factors in determining bad risks is ID fraud(see figure 3). For example, certain characteristics, when combined, prove to be a good predictor of ID fraud. These predictors were found by isolating patterns common to a group of 23 confidence men who caused economic losses in the hundreds of millions of dollars (\$US). However, a sample of 23 confidence men does not support statistically valid predictions about confidence men in general, just as simply being a Palestinian male with a Swiss bank account does not provide any useful information about that individual person's intentions. 
A smarter decision support system would take an individual's past history and measure it against the deviation from the normal population of similar individuals. This can be used to help determine what meaning, if any, this might represent for similar groups of individuals with similar characteristics. We are moving into the need to quantify known and determinable risk factors and to learn from increasingly rich data warehouses/marts. In the midst of social pressures and complex requirements, we need to improve our focus if we are to gain new insights that can be used to make smarter decisions about who constitutes a risks and protect the rights of those who do not.

The data used in credit scoring applications includes ways to identify ID fraud that could be integrated into visa approval processing. Scores can also be integrated with other applications - CRM (Customer Relationship Management); ERP (Enterprise Resource Planning); OLAP (online analytical processing), etc. Most governments are novices in this area, with legislative mandates to protect personal privacy and a need to allocate limited resources among numerous competing priorities. Since it would be unwise to learn on the job where the quality of life for so many is at risk, the governments will need to investigate outsourcing this part of the system development and data processing function. For this reason, there is a rush to absorb the lessons of businesses that have also faced the need to assess risk. Through the use of gap analysis and KITs, in conjunction with competitor profiling (another typical practice of competitive intelligence practitioners), appropriate contractors with the requisite skills can be identified and approached.

\section{$1.4 \quad$ Choosing and Validating Data Mining Models}

As noted earlier, an automated visa risk assessment system can use models to automate those applications (perhaps ten percent) that are clearly going to be approved and some number (perhaps another ten percent) that are clearly going to be denied for cause. By identifying and automating the "rubber stamp" decisions, the analyst can devote more time to exception processing. What the system will identify are the positive (approved) and 
negative (denied) deviations from the norm. Using insights about patterns, an analyst can evaluate the applications that remain (the exceptions) in light of known patterns and associated risks. Not every deviation will be of equal importance. As the outcomes of new decisions feed back into the system, patterns can be refined and used to improve future handling of exceptions. Refining our understanding of patterns that represent some indication of intent to harm, and some means of validating those patterns, must be developed and automated if the system is to do what it is intended to doimprove visa risk assessment.

There are 4 common types of data mining algorithms [Thearling 2002] including (1) k-Nearest Neighbor, which can be used to identify clusters of variables that have predictive value; (2) AI (including CBR discussed below), Genetic Algorithms, and Neural Networks; (3) Rules Induction, and (4) Decision Trees (for example, Bayesian Belief Networks, discussed below). Regardless of the model chosen, it must be evaluated and tested. Each of these models has advantages and disadvantages for use in global risk assessment, and even the best models will not be appropriate for all cases. For this reason, we must also be aware of what the model does not take into consideration.

\subsection{Case-Based Reasoning and Lessons Learned Systems}

One option includes the use of case-based reasoning (CBR) as a framework for developing a visa approval system. CBR is the foundation of Lessons Learned Systems (LLS), in which the application of new insight is used to (1) improve the understanding of the present and (2) improve prediction of future developments. One of the benefits of CBR is that it can walk the analyst through the decision process, eliminating and refining choices so that the most similar cases and lessons learned can be identified and used for comparison. [CBR-Web, 2002]. This option depends on finding ways for the lessons learned from previous results to be embedded into future decisions. With particular reference to the problems of automation of risk assessment systems, research on LLS demonstrates how to learn lessons from both positive and negative project experiences [Verner, Evanco and Weber 2002]. CBR systems enforce a specific problem-solving approach that may not be intuitive to all the analysts who would be expected to use it. Adequate gathering of user requirements is vital if this system is to have any chance of success.

It is important to recognize that the usefulness of CBR depends on access to (and accurate interpretation of) the underlying lessons learned. These lessons are a result of the integration of analysts' decisions and subsequent information that may not appear for some time. For this reason, the successful development and use of LLS is dependent on finding ways to 
model and the accuracy of our interpretation of the resulting score. To set up the scoring process, we first must clean the data. That is, we treat all missing and invalid values and make the known valid values consistent. At this point, we must transform the data. There are two types of data transformation: (1) Data distribution transformation (which involves mathematically altering the distribution of the variable) and (2) Data creation. This type of transformation involves the creation of new variables by combining existing variables to form ratios, differences, and so forth.

For statistical analysis, the data transformation phase is critical because the validity of some statistical methodologies depends on the underlying assumptions that the data is (1) linearly related to an objective variable, (2) normally distributed, and (3) free of outliers. Artificial intelligence, machine learning methods and Bayesian belief networks do not strictly require the data to be normal or linear, and some methods, the decision tree, for example, don't even require dealing with outliers. This is a major difference between statistical analysis and data mining. The machine learning algorithms have the capability to automatically deal with the non-linearity and non-normal distributions (although the algorithms will work better in many cases if these criteria are met). Once the mining is complete, the results can be tested against the data held back (in the vault) to confirm the model's validity. If the model works, its observations should hold for the vaulted data. That is, the model should work well on data it has never seen.

\subsection{Bayesian Belief Networks}

We believe that a visa risk assessment system can benefit from the use of probability-based Bayesian Belief Networks (BBN) (that expand on logistic regression and path analyses), and that this will provide visa analysts with a useful decision support system to assess risks. A BBN consists of a directed acyclic graph, called the network structure, and a set of assessment functions (see figure 5). A directed arc between two nodes in the graph represents a direct influence from the node

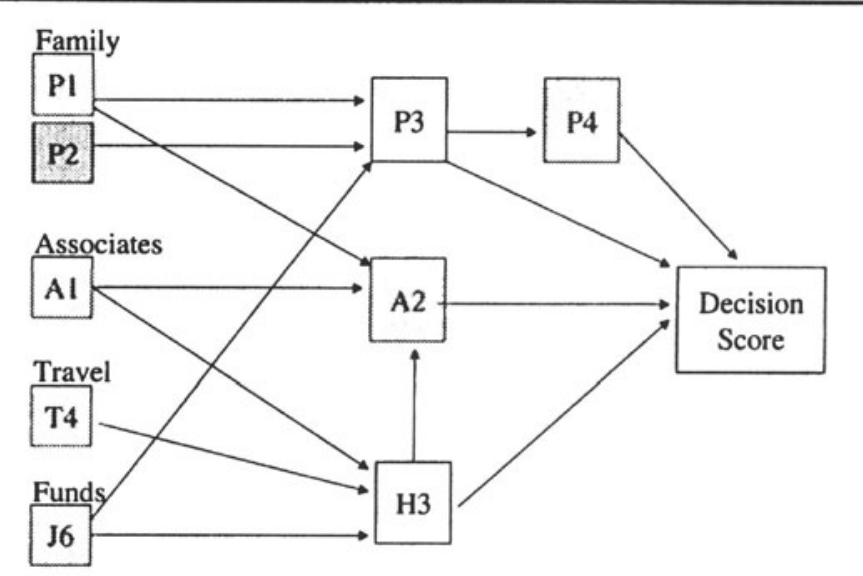

figure 5. Bayesian Belief Networks. 
at the tail to a node at the head. There is one assessment function for each node, defining a conditional probability distribution for the associated variable given the variables that are its parents in the graph. The assessment functions quantify the strength of the dependencies among variables and, together, they define a unique joint probability distribution over the variables, that agrees with the dependencies represented by the network structure. This joint probability distribution represented by a BBN can then be used for decision support through the process of inference. Values of the observed variables can be entered and the probabilities of the other variables can then be computed based on the evidence. The probabilities can be determined on the basis of empirical studies, theoretical insight, or more or less subjective means such as expert opinion. As new information comes in, the probabilities of a BBN are automatically updated to reflect this information [Verner, Evanco and Gefen 2001]. Through the analysis and identification of risk variables, this model will provide visa personnel with perspectives on what risk factors are most critical to visa rejection.

\subsection{Automating a Human Scoring Decision}

In the development of an AVAS, visa analysts would use the output of a data-mining model to further refine the applicant segments that they have specified. The simplest example might involve the segregation of a

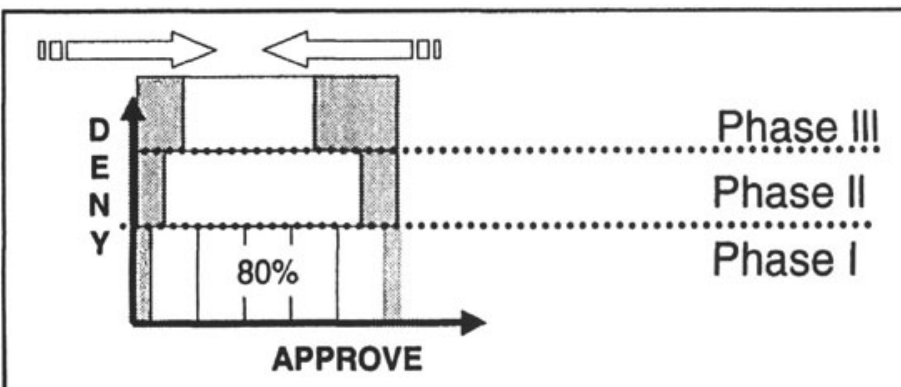

figure 4. Incremental Improvements in Decision Support

group of applicants into separate (rubber stamp) "Approvals/Denials" categories. The data mining could use historical information to build a model of a visa applicant's behavior that could be used to decision support for the AVAS (see figure 4). We could begin with a set of people we know will be cleared/denied. What are their characteristics? What do we know about them? How does risk-based assessment translate into practical applications with relevance for visa approvals, specifically for ID verification [the issue at hand]? Bayesian belief networks can be used to identify changes in relative impact of one variable on another and on clusters of variables.

\subsection{Conclusion}

It should be emphasized that any undertaking of this magnitude requires the integration of data, systems, methods and models form a variety of 
applicant's behavior that could be used to decision support for the AVAS (see figure 4). We could begin with a set of people we know will be cleared/denied. What are their characteristics? What

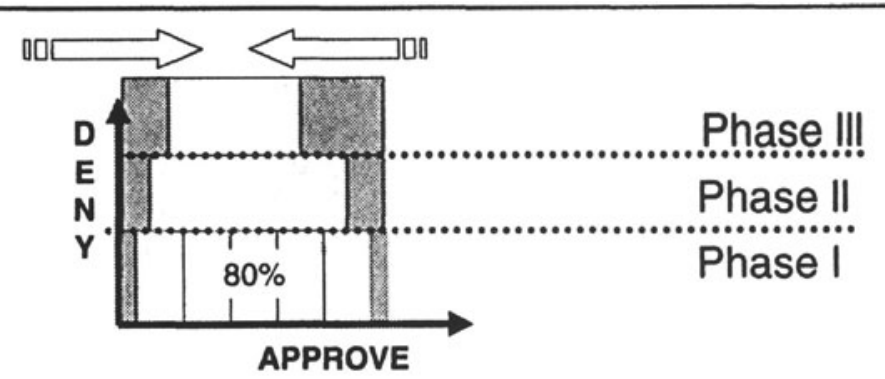

figure 4. Incremental Improvements in Decision Support

do we know about

them? How does risk-based assessment translate into practical applications with relevance for visa approvals, specifically for ID verification [the issue at hand]? Bayesian belief networks can be used to identify changes in relative impact of one variable on another and on clusters of variables.

\subsection{Conclusion}

It should be emphasized that any undertaking of this magnitude requires the integration of data, systems, methods and models form a variety of knowledge domains. The unpalatable truth is that there is an enormous failure rate attached to far less sophisticated software development initiatives [Jones 1994]. Failures may be caused by poor requirements gathering, poor project management, unrealistic schedules, poor change management, and unappreciated (and unmotivated) software development staff who, because of the unrealistic schedules, are expected to work long uncompensated hours that do not register on the radar screens of their managers. The particular difficulty associated with this project is the need to identify data, establish ownership, provide appropriate means of sharing what is relevant, and cleanse the rest. In addition, it may be quite difficult to identify and acquire the necessary algorithmic and data visualizations expertise. Software designers will need a strong background in mathematics/statistics; data visualization expertise in order to present the results in a format that can be easily interpreted by busy visa analysts. Even the testing of such a system will be challenging.

It should also be noted that the models themselves may never do a good job of predicting individual intent to harm, because the intention is a reflection of collateral risks that involve triggering events; for example, the lack of resolution to the Palestinian crisis. Models and scores can only support the aggregation and segregation "highest" and "lowest" risks, but they do free the analyst to spend more time on the thorny problems. The long-term improvements will depend on continuous human intelligence gathering in order to identify new variables of interest and redefine previous variables in light of changing conditions. We need to focus on identifying 
variables that indicate the next most likely threat, rather than the threat that has just passed. For this reason, using both competitive intelligence techniques and an incremental development approach provides the greatest leverage, as new insights can more easily be incorporated into the system as they occur. While the new system should do a much better job than the current system, we must not forget that understanding user requirements and managing customer expectations are important parts of any successful systems development initiative.

\section{REFERENCES}

CBR-Web, the Case-Based Reasoning homepage at the University of Kaiserslautern, http://www.cbr-web.org/.

"Gap Analysis." In CI Strategies and Tools, Intelligence Dictionary, Fuld \& Company, Inc. http://www.fuld.com/dictfiles/record34.html .

Herring, Jan. "Key Intelligence Topics: A Process to Identify and Define Intelligence Needs," CI Review, 1993; 1.2: 4-14.

O’Neill, J. “eGovernment." New York State Archives Technical Series \#58. December 2000. http://www.archives.nysed.gov/pubs/recmgmt/egovernment/definiti.htm.

RiskWise ${ }^{\mathrm{TM}}$ (2002), http://www.riskwise.com/default.html ; and James P. Kervisa, III, quoted in "National Fraud Center Lends Expertise to Economic Crime Summit," LexisNexis" News Release, April-June, 2001.

SCIP. Society of Competitive Intelligence Professionals. http://www.scip.org/ci/.

Shelfer, Katherine M. and Verner, June. "Improving Counterterrorism Analysis: Using Scenarios to support the Development and Use of Integrated Information Systems." Defense Intelligence Journal. 2002; 11. 2: 55-70.

Thearling, Kurt. Introduction to Data Mining. 2002. http://www3.shore.net/ kht/text/dmwhite/dmwhite.shtml.

Verner, June, Evanco, William and Gefen, David, "Modeling Software Develop-ment Risks," Drexel University, unpublished white paper, December 2001.

Verner, June, Evanco, William and Weber, Rosina. "A tool for modeling software development risks, critical success factors and mitigants," Drexel University, unpublished white paper, February 2002.

Verner, June, and Evanco, William. (2002). "A framework for the analysis of the software development process." In Managing Software Engineering Knowledge. Aurum, Aybuke.; Jeffery, Ross.; Wohlin, Claes.; Handzic, Meliha. (Eds) Springer Verlag (in press, to appear late 2002).

Why eGovernment? (2002). New York State Archives, http://www.archives.nysed.gov/pubs/recmgmt/egovernment/whyegovt.htm .

Willox, Norman. Identity Theft: Authentication as a solution. White paper. National Fraud Center, Inc. 2000. http://www.nationalfraud.com/IDENTITY\%20THEFT\%203.13.htm. 